\title{
Tonal Harmony Analysis: a Supervised Sequential Learning Approach
}

\author{
Daniele P. Radicioni and Roberto Esposito \\ Dipartimento di Informatica, Università di Torino \\ \{radicion, esposito\}@di.unito.it
}

November 20, 2008

\begin{abstract}
We have recently presented CarpeDiem, an algorithm that can be used for speeding up the evaluation of Supervised Sequential Learning (SSL) classifiers. CarpeDiem provides impressive time performance gain over the state-of-art Viterbi algorithm when applied to the tonal harmony analysis task. Along with interesting computational features, the algorithm reveals some properties that are of some interest to Cognitive Science and Computer Music. To explore the question whether and to what extent the implemented system is suitable for cognitive modeling, we first elaborate about its design principles, and then assess the quality of the analyses produced. A threefold experimentation reviews the learned weights, the classification errors, and the search space in comparison to the actual problem space; data about these points are reported and discussed.
\end{abstract}

AI in Art and Music; Cognitive Modeling; Machine learning; Music Analysis.

\section{Introduction}

Musical domain always exerted a strong fascination on researchers from very different fields; in the last few years a wealth of research has been invested in attempting to analyze music, under a twofold academic and industrial pressure. Not only music investigation is interesting per se, but it is also required by the novel forms of music e-commerce, e.g. to devise systems for recommendation, algorithmic playlist generation, and music summarization. Recent technological advances significantly enhanced the way automatic environments compose music [1], expressively perform it [2], accompany human musicians [3], and the way music is sold by web stores like iTunes, as well [4].

Music analysis is a necessary step for composing, performing and -ultimatelyunderstanding music, for both human beings and artificial environments (see, e.g., the works in [5] and [6]). Within the broader area of music analysis, we single out the task of tonal harmony analysis. This is a challenging problem for music students, that spend considerable amounts of time in learning tonal harmony, as well as for automatic systems. In Western tonal music at each time point of the musical flow (or vertical) one can determine which chord is sounding: harmonic analysis mainly consists in indicating the fundamental note (or root) and the mode of the chord. 
We have designed a system for tonal analysis, BREVE, where the analysis task is cast to a supervised sequential learning (SSL) problem [7]. From a methodological viewpoint, it transports to the musical domain the state-ofart machine learning conditioned models paradigm, originally devised for the part-of-speech (POS) tagging problem [8]. A set of features has been devised mimicking the main cues used by human experts to analyze music. Intuitively, the analysis of a musical piece can be computed by finding the optimum path in a layered graph (with $T$ layers, one for each vertical) where a node represents one of the possible $K$ labels. The most widely used algorithm to solve this problem is the Viterbi, a dynamic programming algorithm having $\Theta\left(T K^{2}\right)$ time complexity [9]. To overcome the quadratic dependence on $K$, we designed a novel algorithm, CarpeDiem, which finds the optimal path in $O(T K \log (K))$ time in the best case, degrading to Viterbi complexity in the worst case [10]. Further computational considerations about CarpeDiem have been carried out in $[11]$.

In this paper we provide an assessment of the system for tonal harmony analysis. Since our approach puts together various insights from the fields of Machine Learning, Cognitive Science and Computer Music in an interdisciplinary fashion, several aspects are considered. We discuss experiments along three main lines: we elaborate on the meaning of the learned knowledge; we expand on committed errors; and analyze how the search strategy of CarpeDiem allows considering a reduced set of meaningful labels. One major strength lies in the attempt to discuss on the same table strengths and weaknesses of the two cooperating systems: BREVE and CarpeDiem. Yet, an interesting finding is that both accuracies and time performances reveal deep cognitive roots.

\section{Tonal harmony analysis problem}

We define the problem of harmonic analysis as follows. In Western tonal music, at each time point of the musical flow one can determine which chord is sounding. A chord is a set of (three or more) notes sounding at the same time. Harmonic analysis consists in indicating the fundamental note (or root) and the mode of the chord, e.g., C-Maj or F-min, at each time point of the musical flow (Figure 1). Given a score, we individuate sets of simultaneous notes (verticals), and associate to each vertical a label $\langle$ fundamental note, mode $\rangle$. We individuate added notes that can enrich the basic harmony: namely, the cases of seventh, sixth and fourth. This approach to the harmonic structure analysis directly compares, e.g., with that of [12]. However, an higher level functional analysis approach exists that aims at individuating harmonic functions in chords. We'll address the latter in future work.

To analyze the harmonic structure of a piece is a sequential task, where contextual cues play a fundamental role. We start by considering two main musical dimensions: vertical and horizontal information. The former grasps the simultaneity of sounds, whereas the latter applies to successions.

Tonal harmony theory encodes how to build chords, that is which sets of pitches can be played simultaneously. For example, three main kinds or modes of chords are defined, and specifically they are major, minor and diminished chords. Optionally, these basic patterns can be enriched with added notes. This is an example of what we refer to as "vertical" information. Furthermore, tonal 


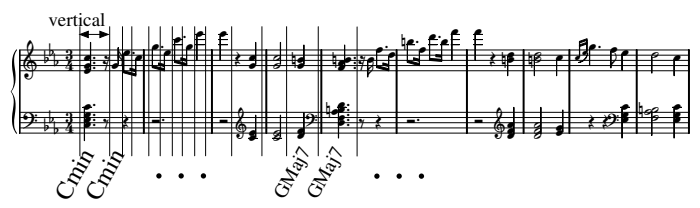

Figure 1: The tonal harmony analysis problem consists of indicating for each vertical which chord is currently sounding. Excerpt from Beethoven's Piano Sonata Op.10 n.1.

harmony theory encodes how to concatenate chords, that is which successions are acceptable: for example, after a $C$-Maj chord one could expect F-Maj or $G$ Maj, rather than $C \sharp-M a j$. This is an example of what we refer to as "horizontal" information.

To complicate things for music analysts, music can be incompletely stated (i.e., we could find only one or two elements of a major triad), or it can be stated by arpeggio (i.e., one note at a time); moreover, passing tones, retards, etc., can make the correct identification a less trivial task. In addition, one has to handle ambiguous cases, where composer's strategies aim at violating listener's expectation: intuitively, this corresponds to breaking "grammatical" rules. To disambiguate unclear cases, analysts refer to horizontal features of music as well. Hence, tonal harmony analysis can be represented as a sequential problem, where both vertical and horizontal aspects prove to be notably influential.

\section{HMPerceptron algorithm grounded on CarpeDiem}

The SSL task can be specified as follows [13]:

Given: A set $L$ of training examples of the form $\left(X_{m}, Y_{m}\right)$, where each $X_{m}=$ $\left(x_{m, 1}, \ldots, x_{m, T_{m}}\right)$ is a sequence of $T_{m}$ feature vectors and each $Y_{m}=\left(y_{m, 1}\right.$, $\left.\ldots, y_{m, T_{m}}\right)$ is a corresponding sequence of class labels, $y \in\{1, \ldots, K\}$.

Find: A classifier $H$ that, given a new sequence $X$ of feature vectors, predicts the corresponding sequence of class labels $Y=H(X)$ accurately.

Presently, $X_{m}$ corresponds to a particular piece of music; $x_{m, t}$ is the information associated to the event at time $t ; y_{m, t}$ is the chord label (i.e., the chord root and mode) associated to the event sounding at time $t$. The problem is, thus, to learn how to predict the sequence of chord labels given the musical events information.

The SSL problem can be solved with several techniques, such as Sliding Windows, Hidden Markov Models, Maximum Entropy Markov Models [14], Conditional Random Fields [15], and Collin's adaptation of the Perceptron algorithm to sequential problems [8] (henceforth, HMPerceptron).

BREVE is implemented by the HMPerceptron algorithm. Although not grounded on a solid probabilistic framework as some of the cited algorithms, the HMPerceptron is reportedly as accurate as other state of the art learning tools, with the advantage of faster learning times. The hypothesis acquired by the HMPerceptron has the form

$$
H(X)=\arg \max _{\bar{Y}=\left\{\bar{y}_{1} \ldots \bar{y}_{T}\right\}} \sum_{t} \sum_{s} w_{s} \phi_{s}\left(X, \bar{y}_{t}, \bar{y}_{t-1}\right)
$$


where $\phi_{s}$ is a boolean function of the sequence of events $X$ and of the previous and current labels. The $\phi_{s}$ functions are called features: they are used by the algorithm to collect information about the salient aspects of the sequence being analyzed. The $w_{s}$ weights are the parameters that need to be estimated by the HMPerceptron. This is done by iterating over the training set and updating the weights so that features correlated to correct outputs are emphasized, whilst those correlated with incorrect ones are penalized. We first introduce the features, and then focus on how the HMPerceptron works.

\subsection{Features design}

In general, features are used to provide discriminative power to the learning system. They are evaluated at each time point $t$ in order to compute an informed prediction about the label to be associated with the event. The present features analyze a small neighborhood of the current event, and return 1 if it contains evidence that the predicted label is correct. Formal definitions cannot be reported here for space reasons. We distinguish among vertical features, that do not require knowledge about the previously predicted label, and horizontal features, that make use of the surrounding context information: both classes are outlined in the following, where $x_{\cdot, t}$ denotes the current event, and $y_{t}$ denotes the currently predicted label.

Vertical features typically report about the presence (or absence) of some note in a given event, thus providing a proof for (or against) a given label. For example, the feature Chord-root-is-asserted informs about whether the root note of label $y_{t}$ is present in event $x_{\cdot, t}$. This feature can provide a precious cue, since the root note is the most salient sound in any chord. Other features providing similar cues are Fully-stated-chord, Asserted-added-note and Root-asserted-innext-event. Altogether, they form the class of features Asserted-degrees.

Features named $v$-chord-notes-asserted are triggered when exactly $\mathrm{v}$ notes of $y_{t}$ are present in $x_{r, t}$. In principle, the smaller is $v$, the lesser evidence exists for $y_{t}$.

It is relevant to point out that our features allow learning classifiers that naturally generalize to unseen chords. For instance, Fully-stated-chord will fire any time all the notes of label $y_{t}$ are present in $x_{, \cdot t}$, regardless of whether $y_{t}$ was present in the training set or not. Such generalization capabilities characterize all features we implemented, and are a major aspect of BREVE.

Horizontal features can be arranged into two classes. One reports about how meter and harmonic changes relate. The other one reports about transitions relevant in tonal harmony theory. Chord-changes-on-metrical-pattern features account for the correlation of label changes and the beat level of a neighborhood of $x_{\cdot, t}$. We have two kinds of beat (accented, unaccented). In analyzing event $x_{\cdot, t}$, we consider events $x_{\cdot, t-1}$ and $x_{\cdot, t+1}$, too. We denote with triplets of 1 and 0 the eight possible metrical patterns. For instance, 010 denotes an accented event surrounded by two weak beats. The associated feature returns 1 if $y_{t-1} \neq y_{t}$ in correspondence of such a pattern.

Human analysts are known to refer to a reduced set of transitions frequent in tonal music. Chord-transition-pattern features are used to model them, as a way for biasing the system to behave accordingly. We represent a transition as a pattern composed by $i$ ) a starting mode and added note; ii) a distance between root notes, expressed in number of half-tones; iii) an ending mode and 
added note. For instance, the notation (Maj7, 5, Maj) refers to a transition from a major chord with added seventh to a major chord with no added note, whose roots are five half-tones apart (e.g., from FMaj7 to BbMaj).

Finally, both Chord-changes-on-metrical-pattern and Chord-transition-pattern features can be used to capture musical aspects that are deeply ingrained with musical style. On one side, this implies that restrictive constraints are posed to the stylistic homogeneity between training and testing sets. On the other one, such style sensitivity grasps valuable 'linguistic' aspects that characterize musical language.

\subsection{Learning Strategy}

As mentioned, to find the best sequence of chord labels for an excerpt can be seen as finding a path in a graph. An example of such a graph for the music analysis problem is reported in Fig. 2.

As a main difference w.r.t. non sequential classification tasks, to classify a sequence implies considering an exponential number of possible label sequences. For instance, in music analysis over 150 different chord labels exist, so that in principle $150^{200}$ possible labelings need to be examined in analysing an excerpt containing 200 events. In order to tame the complexity, a (kind of) first order Markov assumption can be made: this amounts to assume that observing any given label $y_{t}$ does not depend on labels preceding $t-1$. Given the above assumption, Viterbi decoding can be used to decide about the best possible sequence of labels.

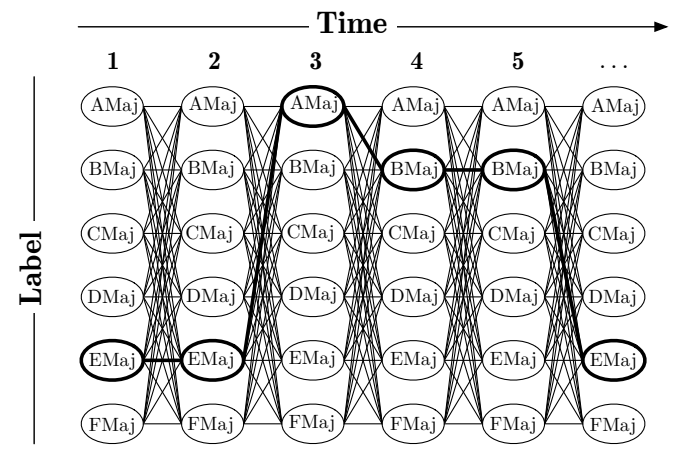

Figure 2: Graphical representation of the labelling problem for $K=6$.

The HMPerceptron algorithm uses Viterbi decoding to evaluate $H$ (see Equation 1) and estimates the weights associated to the features so to maximize $H$ accuracy over the training set. Briefly stated, the algorithm works as follows. The weights vector is initially set to $\mathbf{0}$. Then, for each example (a music excerpt) in the training set, $H$ is evaluated using the current set of weights. If $H$ correctly labels the entire sequence, nothing is done and the algorithm simply jumps to the following sequence. Otherwise, the weights vector is updated so to increment the weights of features that would have contributed to a correct classification, and to decrement the ones that caused a wrong classification. 


\subsection{CarpeDiem algorithm}

As mentioned, it is reasonable to assume that human analysts mainly exploit vertical information, resorting to horizontal cues primarily to resolve ambiguities $[16,17]$. This is to say that to label a given vertical, one first looks at information provided by the current event; then one uses surrounding context to make a decision if still in doubt. Relatedly, vertical information restricts the number of possibilities faced by the analyst, allowing human beings to only consider a subset of possible labeling alternatives.

CarpeDiem implements the described strategy: it uses vertical information over horizontal information, aiming at reducing the number of nodes considered per layer. To provide an intuitive description of the algorithm, it is worth recalling that Viterbi algorithm [9] spends most computational resources to evaluate the formula:

$$
\max _{y_{t}, y_{t-1}}[\overbrace{\omega_{y_{t-1}}}^{\text {weight of the best path to } y_{t-1}}+\overbrace{\sum_{s} w_{s} \phi_{s}\left(X, y_{t}, y_{t-1}, t\right)}^{\text {weight for transition } y_{t-1}, y_{t}}]
$$

where $\omega_{y_{t-1}}$ denotes the weight of the best path to label $y_{t-1}$.

If we partition the set $\{1,2, \ldots p\}$ of all feature indexes into the two sets $\Phi^{0}$ and $\Phi^{1}$, corresponding to indexes of vertical and horizontal features respectively, then the equation (2) can be rewritten into:

$$
\max _{y_{t}}\left[\sum_{s \in \Phi^{0}} w_{s} \phi_{s}\left(X, y_{t}, t\right)+\max _{y_{t-1}}\left[\omega_{y_{t-1}}+\sum_{s \in \Phi^{1}} w_{s} \phi_{s}\left(X, y_{t}, y_{t-1}, t\right)\right]\right]
$$

Equation (3) is equivalent to Equation (2); in addition, it emphasizes how features in $\Phi^{0}$ need to be evaluated only once per $y_{t}$ label, and not once for each $y_{t}, y_{t-1}$ pair. It is then obvious (and in accord with the intuition) that whenever $\Phi^{1}=\emptyset$ the cost of the Viterbi algorithm can be reduced to be linear in the number of labels. The core idea underlying CarpeDiem is to exploit vertical information for avoiding the evaluation of the inner maximization as long as possible. In this way, we resort to the use of horizontal features only in case this is really necessary for the classification purposes.

\section{Experimentation}

In previous works we showed that BREVE accuracy is $81.5 \%$ on a corpus of Bach chorales; this figure lowers to $73.8 \%$ over a much less homogeneous dataset (namely, the Kostka-Payne corpus [18]). Also, CarpeDiem saves over $88 \%$ of classification time w.r.t. Viterbi [11]. Here we are concerned with the quality of its outcomes and, specifically, we explore $i$ ) whether the learned rules are meaningful, based on musical accounts; $i i)$ whether the error analysis can shed any light or suggest any improvement to the set of features or to the classification strategy, and iii) whether the labels actually considered through the search process are to some extent similar to the 'reduced set' of labels considered by humans. 
The system has been trained on a data set composed of 30 4-parts harmonized chorales by J.S. Bach. A different set of 42 chorales from the same author has been used for testing.

i) Did we learn anything musically meaningful? In Figure 3 we present the learned weights arranged into the four classes introduced above.

The highest positive weights involve vertical features (see for instance Chordroot-is-asserted (Fig. 3(a)) and v-chord-notes-asserted (Fig. 3(b))): as expected, information about which notes are currently sounding prevails over contextual information. In other terms BREVE, like human analysts, puts much emphasis on vertical information ${ }^{1}$. In considering $v$-chord-notes-asserted features (Fig. $3(\mathrm{a}))$, we see that the case $v=3$ received more emphasis with respect to the case $v=4$. In the examined corpus events with 4 or more notes are likely to contain passing tones which mislead the 4-chord-notes-asserted feature. Furthermore, the lower frequency with which the feature is asserted influences the magnitude of the learned weight as well.

In cases $v=1$ and $v=2$ we note how the features are used to report about evidence against a given label, rather than supporting it. We also note how the feature with $v=1$ received a penalty heavier than the feature with $v=2$. This in spite of the fact that seeing one single asserted note provides, in principle, much more evidence against a given label w.r.t. seeing two of them. To understand this surprising fact, one needs to recall that the HMPerceptron changes the weight of a given feature when the predicted labeling is wrong. In particular, the algorithm decreases the weight of the features that voted for the wrong class, and increases the weight of features that voted for the correct one. 1-chord-notes-asserted feature fires when harmony is only loosely stated. If, in the meanwhile, the correct label does contain exactly one of the sounding notes, then the feature correlates with exact classification and its weight is augmented. On the contrary, when more notes are present and the classification is wrong, the label predicted by HMPerceptron probably contains more than a single note in common with the current vertical. Thus, the feature for $v=1$ is not to be deemed responsible for the error and its weight is not decremented. The same explanation, with inverted arguments holds for $v=2$, since this feature is the most likely to fire in correspondence of wrong labelings.

By looking at the Asserted-degrees features class (Fig. 3(b)), we observe that the features Fully-stated-chord, Chord-root-is-asserted, and (2|3)-chord-notes-asserted necessarily fire simultaneously. In such situations, the 'amount of evidence' in favour of $y_{t}$ is overwhelming and BREVE will probably investigate only few alternative labels.

Let us now consider horizontal features (Figure 3(c) and 3(d)). Weights associated to harmony changes in correspondence with weak beats (?0? patterns) receive the highest penalty among all features. While harmony change is globally discouraged, the system clearly exhibits a preference for changing harmony on accented beats. The preference for chord transitions in correspondence with accented events substantially fits to experimental evidences and analytical strategies known in music cognition literature, e.g., the Strong beat rule proposed by [16], and experimentally verified by Temperley [17]. Interestingly, the speed of harmony changes is deeply ingrained with musical style: these features

\footnotetext{
${ }^{1}$ It is also interesting to study the musical and cognitive effects of weights' evolution in a dynamic fashion, as learning proceeds. The surprising computational impact of weights evolution on classification times has been investigated in [11].
} 


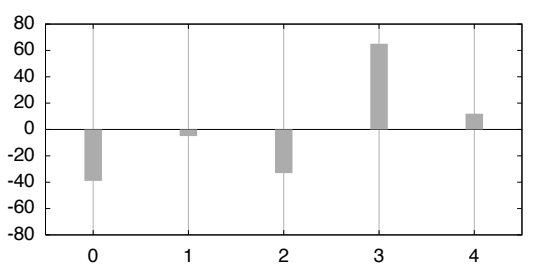

(a) $v$-chord-notes-asserted features

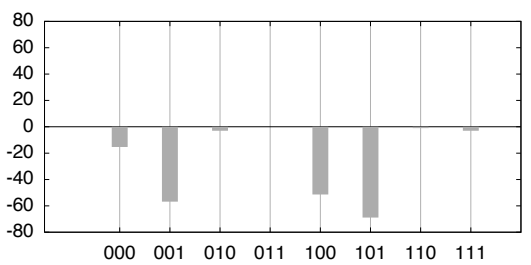

(c) Change-vs-meter features

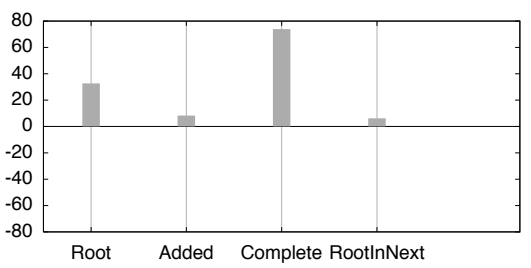

(b) Asserted-degrees features

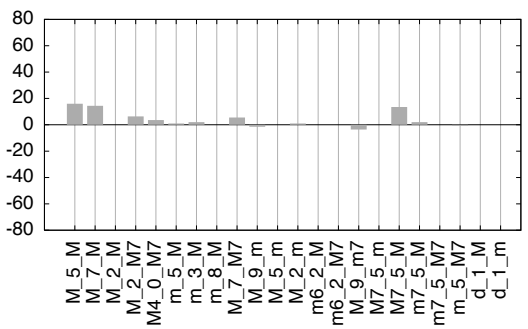

(d) Chord-transitions features

Figure 3: Weights learned on a training set composed by 30 4-parts harmonized chorales by J.S. Bach. Graphics in the first row present weights obtained by vertical features, whilst in the second row the weights obtained by horizontal features are presented.

result in a rough way of modeling the harmonic rhythm and, as a consequence, they provide important indications about style.

Chord-transition-pattern features do not provide highly discriminative contributions to the classification process; they rather constitute refinement criteria when classification is ambiguous. This fact is somehow surprising, as in Bach chorales one would expect to find few, well-distinguished transition patterns. However, each and every transition in the cadence have been identified ${ }^{2}$. In fact, the following five best scoring features are those needed to build cadences.

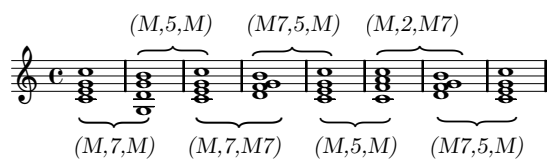

This fact would suggest that (at least for dealing with Bach chorales), it is possible to reduce the Chord-transition-pattern features to those involved in the cadence, in that all the other ones are mainly used for fine tuning purposes. A new experimentation with the Chord-transition-pattern features reduced to the five mentioned still obtains a $79.44 \%$ classification accuracy $(2 \%$ less than with the full block).

ii) Do errors shed any light on the system's functioning, or suggest any improvement? As mentioned, BREVE is incorrect in the $18.5 \%$ of cases.

\footnotetext{
${ }^{2}$ Cadence is a sequence of chords comprising the close of a musical phrase, which is commonly acknowledged to be a sort of "cradle" of tonal language.
} 
A closer look at the errors shows some identifiable error classes. First, in about $30 \%$ of errors, we obtain completely wrong labelings. In these cases BREVE classifies with wrong root, wrong mode and wrong added note: i.e., situations where the system is totally mislead. In many cases the system is caught in interpretative nuances that are hardly avoided given the simplified input representation (e.g., the system cannot consider inversions, chord substitutions, nor tonality). In other cases, the resistance to chord changes forces the system to inherit by mistake previously attributed labels (this is especially true for chord changes on weak beats).

Among other mistakes, few errors appear execrable from the viewpoint of harmony theory. For instance, those due to confusing chord modes such as major with minor chords or viceversa. This is an error never committed by human analysts, and seldom committed by BREVE: only $5 \%$ of the overall error rate falls in this class. One may argue that a lager context is needed to improve on this issue.

The errors due to confusion between relative keys amount to $15 \%$ of the total. Relative keys are intrinsically related tones (in that they share the same key signature while having different roots), and to confuse between them is considered venial from a musical perspective.

Many errors (namely the $23.2 \%$ ) are due to wrong added note (root and mode being correct). In these cases the chord is analyzed in a substantially correct fashion. In some cases, it is again the resistance to chord changes that causes this kind of mistake. For example, in the case of succession $F-M a j \rightarrow F-$ $M a j \rightarrow B b-M a j$ let us consider the case that the added seventh of $F$-Maj is stated in correspondence of the second unaccented F-Maj chord. This case can be controversial even for human analysts, in that both F-Maj and F-Maj7 can be considered correct labelings for the second vertical. In summary, there is evidence that the system makes errors that are either venial, or justifiable on musical accounts, or due to information lacking in the input representation.

iii) Which labels are considered. On average, BREVE inspects only a fraction of all possible labels. This datum, which grasps a kind of "computational effort" measure, correlates (though only mildly) with the classification error. In fact, if we consider the correctly predicted labels, BREVE inspects $48 \%$ of them; this figure raises to $62.5 \%$ in case of wrong predictions. In other words, to analyze difficult events a larger effort is required. We now show that inspecting few/more labels obeys a musically reasonable strategy.

Let us examine the behavior of BREVE in action as it analyses the first few measures from the chorale presented in the top part of Figure 4. We consider this toy example (many more labels are usually present) suitable to provide some insight on BREVE's inner mechanisms. At the bottom of the Figure we report three pictures showing the state of the analysis (i.e., nodes just inspected (opened), nodes yet to be opened, and nodes opened in previous steps), at the time points $t=7,8,9$.

BREVE always inspects all nodes of the first vertical: only for the first time step this is a cheap operation that through a preliminary reconnaissance provides solid ground for subsequent investigation. In the subsequent steps, BREVE exploits vertical information to inspect only few labels.

Figure 4 points out a that counterintuitive fact occurs at $t=8$. Even though ambiguities arise at time step 8, to solve them the algorithm inspects 16 labels for event 7 , and only 3 for event 8 . At $t=8$ we have a passing note on a 


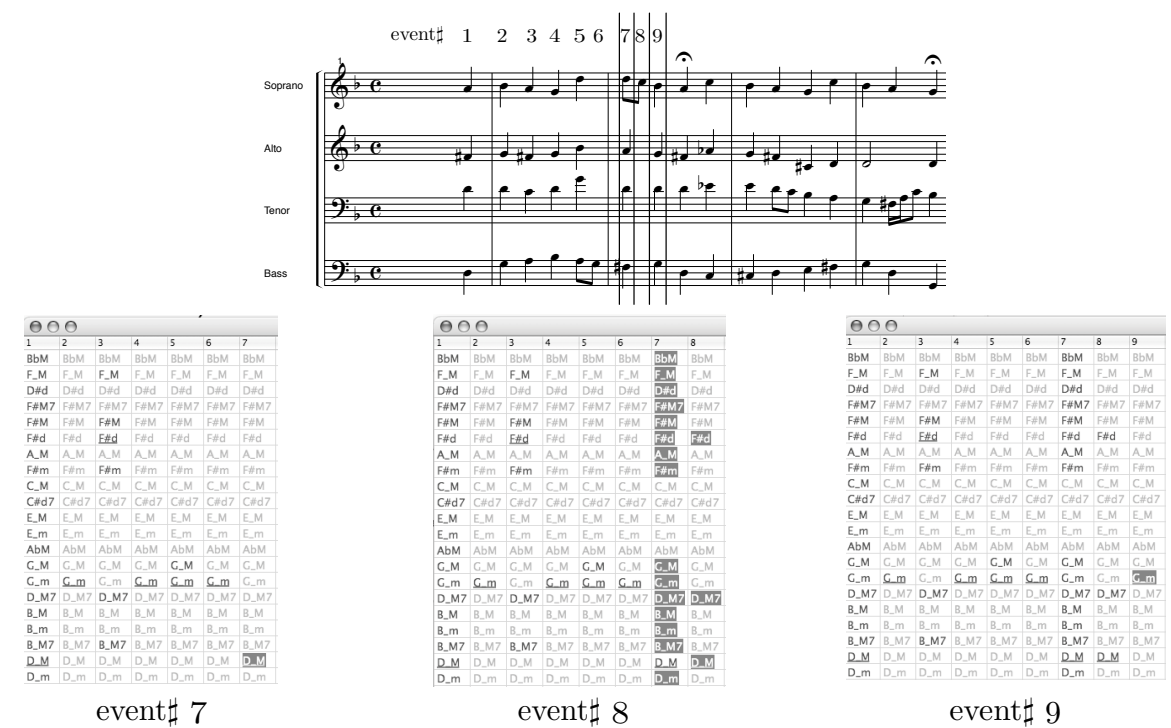

Figure 4: The starting measures of the chorale $B W V 206$ by J.S. Bach (top), along with three dumps of the analysis graph when dealing with the events at times $t=7,8,9$ (bottom). Gray chord labels indicate nodes yet to be inspected (closed), while black labels stand for open nodes; white labels on a gray background indicate nodes that have been opened at the last step; finally, underlined labels indicate the correct label.

weak beat; notes sounding at $t=8\langle F \sharp, D, A, C\rangle$, make labels $D$-Maj, D-Maj7 and $F \sharp$-dim plausible. It is then necessary to find the best ancestors for those nodes in the $7^{\text {th }}$ layer, thus considering their 'true' horizontal contribution to the maximization strategy (see [10]). Among the possible ancestors considered at time step 7 there are some that are not completely justifiable on musical accounts. BREVE often succeeds in reducing the search space by exploiting a bound on the maximal horizontal score. Instead, in the present case the bound is not sufficient to cut the search and the algorithm forcefully inspects even less musically justifiable alternatives like B-Maj. It is somehow ironic that, after all the efforts spent in analyzing this layer, metric considerations override any other criterion and prevent harmony from changing. Though correct, this is amazing, in that D-Maj7 chord is fully asserted, and it is remarkable how much horizontal features contribute to tune the classification towards meaningful labelings.

\section{Conclusions}

This paper addressed the issue of tonal harmony analysis, which is at the ridge of AI, Cognitive Science and Computer Music. After surveying the learning principles of the HMPerceptron algorithm, we have pointed out that music harmony is an appropriate test-bed for SSL classifiers. The classifiers designed for tonal analysis greatly benefit from working in conjunction with CarpeDiem. In addition to computational gain w.r.t. to the Viterbi algorithm, we argued that CarpeDiem performs analyses that are also more justifiable on cognitive grounds. Results have been presented about a threefold experimentation discussing $i$ ) the 
meaning of the learned weights -interpretable as high-level rules-; $i i)$ which errors were committed -along with some arguments on causes and solutions-; and iii) the part of the search space actually explored.

Few final remarks about the future extensions of the work are in order. First, we will focus on extending the music representation to allow taking into account inversions, chords substitutions and functional analysis. Moreover, we are devising an extension of CarpeDiem that allows for the exploitation of higher order Markovian hypotheses.

\section{References}

[1] Cope, D.: A Musical Learning Algorithm. Comput. Music J. 28(3) (2004) $12-27$

[2] Grachten, M., Arcos, J.L., López de Mantaras, R.: A Case Based Approach to Expressivity-Aware Tempo Transformation. Mach. Learn. 65(23) (2006) 411-437

[3] Raphael, C.: A Hybrid Graphical Model for Rhytmic Parsing. Artif. Intell. 137(1-2) (2002) 217-238

[4] Freeman, J.: Fast generation of audio signatures to describe iTunes libraries. Journal of New Music Research 35(1) (2006) 51-61

[5] Widmer, G.: Discovering Simple Rules in Complex Data: A Meta-learning Algorithm and Some Surprising Musical Discoveries. Artif. Intell. 146 (2001) 129-148

[6] López de Mantaras, R., Arcos, J.: AI and Music: From Composition to Expressive Performance. AI Magazine 23 (2002) 43-57

[7] Radicioni, D.P., Esposito, R.: A Conditional Model for Tonal Analysis. In Esposito, F., Ras, Z.W., eds.: Foundations of Intelligent Systems. Number 4203 in LNAI, Berlin, Springer-Verlag (2006) 652-661

[8] Collins, M.: Discriminative training methods for hidden markov models: Theory and experiments with perceptron algorithms. In: Proceedings of the Conference on Empirical Methods in Natural Language Processing. (2002)

[9] Viterbi, A.J.: Error Bounds for Convolutional Codes and an Asymptotically Optimum Decoding Algorithm. In: IEEE Transactions on Information Theory. Volume 13. (1967) 260-269

[10] Esposito, R., Radicioni, D.P.: CarpeDiem: an Algorithm for the Fast Evaluation of SSL Classifiers. In: Proceedings of the 24th Annual International Conference on Machine Learning (ICML 2007). (2007)

[11] Esposito, R., Radicioni, D.P.: Trip Around the HMPerceptron Algorithm: Empirical Findings and Theoretical Tenets. In Pazienza, M.T., Basili, R., eds.: AI*IA 2007: Advances in Artificial Intelligence, 10th Congress of the Italian Association for Artificial Intelligence, Springer-Verlag (2007) 
[12] Pardo, B., Birmingham, W.P.: Algorithms for chordal analysis. Comput. Music J. 26 (2002) 27-49

[13] Dietterich, T.G., Ashenfelter, A., Bulatov, Y.: Training conditional random fields via gradient tree boosting. In: ICML '04: Twenty-first international conference on Machine learning, New York, NY, USA, ACM Press (2004)

[14] McCallum, A., Freitag, D., Pereira, F.: Maximum entropy Markov models for information extraction and segmentation. In: Proc. 17th International Conf. on Machine Learning, Morgan Kaufmann, San Francisco, CA (2000) $591-598$

[15] Lafferty, J., Pereira, F.: Conditional random fields: Probabilistic models for segmenting and labeling sequence data. In: Procs. of the 18th International Conference on Machine Learning, San Francisco, CA, Morgan Kaufmann (2001) 282-289

[16] Lerdahl, F., Jackendoff, R.: A Generative Theory of Tonal Music. MIT Press, Cambridge, MA (1983)

[17] Temperley, D.: The Cognition of Basic Musical Structures. MIT, Cambridge, MASS (2001)

[18] Kostka, S., Payne, D.: Tonal Harmony. McGraw-Hill, New York, NY (1984) 